\title{
Ketamina en el manejo del dolor refractario por cáncer
}

\author{
Karla Sofía Vargas Rumoroso, Isaías Salas Herrera
}

\section{Resumen}

Los pacientes con cáncer presentan dolor en el rango entre moderado e intenso durante el transcurso de su enfermedad, principalmente en la etapa terminal. La escalera analgésica de la Organización Mundial de la Salud (OMS) se ha empleado como guía terapéutica para tratar a estos pacientes; utiliza tres grupos de fármacos: opiáceos, antiinflamatorios no esteroideos (AINES) y coadyuvantes. Dentro de estos últimos se encuentra la ketamina, un derivado fenciclidínico que interactúa con los receptores N-metil-D-aspartato (NMDA). Tradicionalmente ha sido utilizado como inductor de la anestesia general, y en dosis subanestésicas se emplea para el tratamiento del dolor refractario al uso de opioides.

Descriptores: dolor, cáncer, analgesia, ketamina

Key words: pain, cancer, analgesia, ketamine

Recibido: 28 de octubre de 2005

Aceptado: 17 de enero de 2006

Centro Nacional de Control del Dolor y Cuidados Paliativos

\begin{abstract}
Abreviaturas: AINES: Antiinflamatorios no esteroideos; NMDA, NMetil-D-Aspartato; OMS, Organización Mundial de la Salud; PCP, Fenciclidina, SNC, Sistema Nervioso Central.
\end{abstract}

Correspondencia: Karla Sofía Vargas Rumoroso. E-mail:

ksvargasI@hotmail.com Isaías Salas Herrera. E-mail: isaiassalas@racsa.co.cr

ISSN 0001-6002/2006/48/2/61-65 Acta Médica Costarricense, @2006 Colegio de Médicos y Cirujanos
El dolor por cáncer es un problema muy importante de salud pública, pero olvidado por las autoridades. La mayor parte de los pacientes oncológicos en los países en desa-rrollo no reciben cuidados paliativos adecuados ${ }^{1}$. En sus inicios, el dolor por cáncer se puede presentar en un $30 \%$ de los pacientes, y en su etapa final hasta en un 80 ó $90 \%{ }^{2}$.

El fundamento para el uso de la ketamina en el control del dolor y cuidados paliativos inicia muchos años atrás, cuando se utilizaba como complemento de la anestesia regional para procedimientos quirúrgicos, como bloqueo de nervios, anestesia espinal o epidural y control del dolor en curaciones de pacientes quemados ${ }^{3,4}$. Recientemente, dosis bajas de ketamina (250 $\mu \mathrm{g} / \mathrm{Kg}$ ) se han usado efectivamente para el control del dolor postoperatorio refractario a opioides $^{5-8}$. Con el uso de dosis bajas de ketamina en pacientes terminales con dolor refractario al tratamiento farmacológico convencional, las dosis de opioides y coadyuvantes (gabapentina) se disminuyeron en un $50 \%$, por lo que se trata de una opción terapéutica disponible para controlar el dolor por cáncer ${ }^{9}$.

El objetivo de este artículo es llevar a cabo una revisión de la bibliografía médica con el propósito de determinar la efectividad del uso de ketamina en el dolor refractario por cáncer.

\section{Farmacología}

El nombre químico de la ketamina es 2-(2-clorofenil)-2-(metilamino) ciclohexanona. Su principal efecto se produce en el receptor N-metil-D-aspartato (NMDA), a nivel central, y en la sustancia gelatinosa de la médula espinal ${ }^{10,11}$, (el mismo receptor de la fenciclidina [PCP]), lo 
que genera un antagonismo no competitivo de dicho receptor.

Actúa sobre múltiples sistemas neurotransmisores, como noradrenalina, serotonina y colinérgicos muscarínicos del SNC. Además, tiene una acción aún poco conocida, sobre el receptor opiáceo sigma. En dosis de inducción anestésica provoca un aumento de la presión intracraneana ${ }^{12}$, intraocular, pulmonar y arterial, y aumenta la frecuencia cardiaca.

Su vía de eliminación es hepática: se metaboliza en norketamina (metabolito activo y mayor productor de la analgesia ${ }^{13}$ ) y después en 6-hidroxi-norketamina, metabolito inactivo excretado por los riñones.

La ketamina administrada vía oral produce un pico de concentración sanguínea a los 30 minutos, seguido de otro pico de concentración de su metabolito activo, norketamina, a la hora, la cual se mantendrá detectable en sangre hasta 7 horas después de su administración ${ }^{14}$.

Por tanto, la ketamina actúa de dos maneras. La primera, revirtiendo la tolerancia a la morfina y la hiperalgesia producida por opioides (efecto antihiperalgésico) y la segunda, como antagonista de los receptores NMDA. Esta función ha sido relacionada con la reducción de la intensidad del dolor (efecto antinociceptivo).

\section{Análisis de la bibliografía}

Las pruebas provenientes de modelos animales experimentales, estudios en voluntarios humanos y ensayos clínicos, indican que las dosis subanestésicas de ketamina en diferentes vías de administración alivian diversos síndromes dolorosos crónicos y neuropáticos ${ }^{15-17}$. Además, cumple un papel en la prevención del desarrollo de la tolerancia a los opiáceos, ya que en un modelo experimental con ratas se demostró su capacidad para prevenir la hiperalgesia inducida por opiodes ${ }^{18} \mathrm{y}$ la posterior tolerancia aguda a la morfina ${ }^{19-21}$. Es por ello que la ketamina se ha estado utilizando para el tratamiento del dolor refractario en cáncer ${ }^{22,23,24,25}$. En adición, el costo, relativamente bajo, hace apropiado su uso en países subdesarrollados ${ }^{26}$.

Thogulava et al, en un estudio de 9 pacientes con dolor neuropático secundario a cáncer, con dosis máximas de opioides y otros coadyuvantes, indicaron que la ketamina oral en el tratamiento de plexopatía maligna podría ser un potente analgésico en dosis de $0.5 \mathrm{mg} / \mathrm{Kg}$. de peso corporal, administradas tres veces al día, y que goza de un perfil más favorable en cuanto a sus efectos secundarios, que la ketamina parenteral ${ }^{27}$. Kannan et al utilizaron ketamina en la misma dosis, tres veces al día, en combinación con morfina oral, para el tratamiento de dolor neuropático maligno en nueve pacientes. Siete de ellos reportaron una disminución de hasta tres puntos en la escala de dolor ${ }^{28}$. Friedman et al, en un reporte de caso de un paciente de 59 años con hepatocarcinoma secundario a hepatitis $\mathrm{C}$, que no respondía a la terapia con opioides, señalan que, tras administrarle $30 \mathrm{mg}$ VO de ketamina, en menos de 30 minutos desapareció el dolor y su estado mental (medido por minimental score) regresó al basal ${ }^{29}$. McDonnell et al, en un estudio de orden observacional realizado a humanos voluntarios con dolor por cáncer, sugieren que la ketamina oral posee un efecto analgésico más potente que la ketamina parenteral y produce menos efectos secundarios ${ }^{30}$.

Por otro lado, en un estudio multicéntrico realizado por Jackson et al a 39 pacientes con dolor somático y neuropático refractario por cáncer, se indica que una terapia breve de infusión subcutánea continua de ketamina es efectiva, en una dosis inicial de $100 \mathrm{mg}$ por día, que puede incrementarse a $300 \mathrm{mg}$, hasta una dosis máxima de $500 \mathrm{mg}$ diarios si el control del dolor no es adecuado ${ }^{10}$. El tratamiento se continuó por un lapso de 3 a 5 días, utilizando la menor dosis eficaz, o una dosis máxima de $500 \mathrm{mg}$ diarios. Los resultados fueron que el $88 \%$ de los pacientes con dolor somático y el $61 \%$ con dolor neuropático respondieron satisfactoriamente, e incluso el $83 \%$ mantuvieron niveles adecuados de dolor durante las ocho semanas siguientes a la suspensión del tratamiento. En otro reporte de caso publicado por Bam et al referente a una niña de 2 años de edad con dolor refractario secundario a neuroblastoma metastático, se refiere que se le administró una infusión intravenosa de ketamina, y que 24 horas posterior a esta era manifiesto un adecuado control del dolor y una mejoría de la capacidad cognitiva ${ }^{31}$. En estudios efectuados por Mercadante et al a 10 pacientes con dolor neuropático por cáncer refractario al uso de opioides, la ketamina parenteral en forma de bolo intravenoso en dosis de $0.25-0.5 \mathrm{mg} / \mathrm{Kg}$. disminuyó de forma significativa la intensidad del dolor en casi todos los pacientes ${ }^{32}$. Yang, en un reporte de caso de una paciente de 77 años con cáncer de recto con metástasis vertebrales e infiltración del plexo sacro, inició con esquemas de medicamentos vía oral y opioides transdérmicos y debido a falla en control del dolor, se le colocó un catéter espinal con infusión de morfina y bupivacaína, sin resultados satisfactorios. Se agregó ketamina 1,0 $\mathrm{mg} / \mathrm{kg}$ por vía intratecal, la cual redujo la dosis intratecal de morfina requerida para controlar el dolor por cáncer y casi eliminó su dolor neuropático. Se administró por espacio de 21 días, hasta el fallecimiento de la paciente ${ }^{33}$.

Carr et al, en un estudio doble ciego con 20 pacientes con dolor crónico por cáncer utilizaron ketamina intranasal vs. placebo. Quienes usaron ketamina intranasal (10 mg spray máximo \#5 por día) obtuvieron mayor alivio de sus síntomas, en comparación con aquellos que emplearon placebo, con inicio de la analgesia en 10 minutos y duración del efecto por 60 minutos ${ }^{34}$. 
Por último, Gammaitoni et al realizaron un estudio en cinco pacientes con dolor neuropático resistente a opioides y utilizaron ketamina tópica, presentación en gel, en dosis de $0.093 \mathrm{mg} / \mathrm{kg}$ a $9.33 \mathrm{mg} / \mathrm{kg}$, dependiendo del área de superficie y la zona de la aplicación. Todos los pacientes percibieron disminución significativa del dolor después de quince minutos de la aplicación. Los autores recomiendan la utilización de la ketamina en gel solo cuando se han agotado todas las medidas de primera y segunda opción ${ }^{35}$.

\section{Efectos adversos}

En su estudio, Mercadante encontró que tres pacientes experimentaron alucinaciones cuando recibieron ketamina $0,25 \mathrm{mg} / \mathrm{Kg}$. y ketamina $0,5 \mathrm{mg} / \mathrm{kg}^{32}$. Pacientes con niveles séricos de ketamina menores a 50 microgramos por mililitro no las desarrollaron. Jackson debió disminuir la dosis de ketamina en 5 pacientes debido a efectos psicomiméticos considerables. Ellos también experimentaron flashes de luz, una sensación de "zumbido" en la cabeza y de falta de sobriedad ${ }^{10}$. Según Mercadante, el diazepam resolvió estos síntomas en dosis de 1 mg IV. Además, Rae asoció aumento en la somnolencia cuando se utilizaba ketamina junto con morfina, lo cual es un efecto dosis dependiente ${ }^{22}$. Otros efectos adversos menos comunes fueron: náuseas, vómitos, boca seca y confusión. Con respecto a la ketamina intranasal, el único efecto secundario reportado fue, en dos pacientes, elevaciones transitorias de la presión arterial ${ }^{34}$.

\section{Contraindicaciones}

Debe evitarse en pacientes con hipersensibilidad conocida a la ketamina y en quienes padezcan hipertensión endocraneana, y utilizarse con precaución en los epilépticos ${ }^{36}$.

\section{Protocolo para el uso de ketamina}

El Dr. Perry Fine, anestesiólogo y especialista en medicina paliativa de la Universidad de Utah, ha desarrollado el siguiente protocolo para el control del dolor refractario por cáncer, en cuidado paliativo, con resultados satisfactorios durante los últimos años:

1- Ketamina en bolo en dosis de 0.1-0.2 mg/Kg. IV ó 0.5 $\mathrm{mg} / \mathrm{Kg}$. SC o IM. (Idealmente, utilizar el acceso intravenoso, por la rapidez y efectividad para titular las dosis del fármaco.)
Asegurar monitoreo de signos vitales y escala del dolor. Explicar a los pacientes la posibilidad de experimentar alucinaciones, en especial a aquellos que no son capaces de comunicarse adecuadamente debido a su enfermedad.

2- Si tras 15 minutos de la aplicación de la dosis IV, ó 3545 minutos después de la dosis SC o IM no hay disminución significativa del dolor, utilizar el doble de la dosis de ketamina indicada. Continuar el monitoreo y el doblaje de las dosis hasta que haya disminución significativa del dolor o aparición de efectos secundarios indeseables, como aumento de las secreciones (contrarrestado con el uso de un antisialogogo del tipo glucopirrolato, escopolamina o atropina) o sensaciones disfóricas (contrarrestadas por el uso de benzodiacepinas como diazepam o lorazepam o fenotiazidas/butirofenonas como clorpromacina o haloperidol).

3- Calcular la dosis total administrada y la duración de su efecto e iniciar una infusión continua IV o SC determinada horariamente y calculada de la siguiente manera: dosis total administrada / duración estimada del efecto $=$ velocidad de infusión en $\mathrm{mg} / \mathrm{hr}$. Monitorear y ajustar la velocidad de la infusión, según los signos y síntomas del paciente.

4- Disminuir en un $50 \%$ la dosis del opioide utilizado y disminuirla a la mitad cada 6-12 hrs., según lo tolerado por el paciente. Tener listo un bolo de opioide, por si aparecen síntomas de abstinencia. Aplicar nuevos bolos de ketamina e incrementar la dosis de la infusión si es necesario, solo hasta la aparición de efectos psicomiméticos desagradables.

\section{Conclusiones}

La ketamina podría ser una coadyuvante muy efectiva para el control del dolor refractario al tratamiento farmacológico convencional. Su riesgo es bajo, su eficacia es alta y se trata de una droga relativamente barata con resultados gratificantes.

Los estudios clínicos actuales demuestran que la ketamina puede ser una buena opción de tratamiento coadyuvante si se administra temprano en la enfermedad, ya que disminuye el fenómeno "wind-up" (sensibilización central). Sin embargo, se requieren más estudios clínicos, muestras homogéneas y de mayor tamaño, que permitan ofrecer conclusiones convincentes sobre este medicamento. 


\section{Abstract}

Patients with cancer suffer moderate to severe pain during the course of their disease, mainly during the terminal phase. The World Health Organization Pain Relief Ladder has been used as a therapeutic guide for the treatment of these patients, which uses 3 kinds of drugs: opioids, non steroidal anti-inflammatory drugs, and adjuvants. Ketamine is one of the latter; it is a phencyclidine derivative that interacts with N-methyl-D-aspartate (NMDA) receptors. Traditionally it has been used as an anesthesia induction drug, and subanesthetic doses are used for relief of pain refractory to opioids.

\section{Referencias}

1. Organización Mundial de la Salud. National cancer control programmes: policies and manage trial guidelines. Ginebra, 2002.

2. Organización Mundial de la Salud. Adherencia a los tratamientos a largo plazo: pruebas para la acción. Ginebra, 2003.

3. Fine P. Low-dose ketamine in the management of opioid resistant terminal cancer pain. J Pain Symptom Manage. 1999;17:296-300.

4. Patterson D, Shahar S. Burn pain. In: Loeser J, et al., editors. Bonica's management of pain. Philadelphia: Lippincott, Williams and Wilkins; 2001. pp. 781-7.

5. Weinbroum A. A single small dose of postoperative ketamine provides rapid and sustained improvement in morphine analgesia in the presence of morphine resistant pain. Anesth Analg. 2003;96:789-95.

6. Bell R, Dahl J, Moore R, Kalso E. Peri-operative ketamine for acute post-operative pain: a quantitative and qualitative systematic review Acta Anaesthesiol Scand. 2005;49:1405-28.

7. Kafali H, Aldemir B, Kaygusuz K, Gursoy S, Kunt N. Small-dose ketamine decreases postoperative morphine requirements. Eur J Anaesthesiol. 2004 Nov;21:916-7.

8. Fine P. Ketamine: From Anesthesia to Palliative Care. AAHPM Bulletin 2003, 3: 1-6.

9. Richebe P, Rivat C, Laulin JP, Maurette P, Simonnet G. Ketamine improves the management of exaggerated postoperative pain observed in perioperative fentanyl-treated rats. Anesthesiology. 2005; 102:421-8.

10. Jackson K, Ashby M, Martin P.: "Burst" Ketamine for refractory cancer pain: an open-label audit of 39 patients. J Pain Symptom Manage 2001; 22: 834-42.

11. Pasero C, McCaffery M. Pain control: ketamine: low doses may provide relief for some painful conditions. Am J Nurs. 2005;105:60-4.

12. Langsjo J, Maksimow A, Salmi E, Kaisti K, Aalto S, Oikonen V, Hinkka S, Aantaa R, Sipila H, Viljanen T, Parkkola R, Scheinin H. Sketamine anesthesia increases cerebral blood flow in excess of the metabolic needs in humans. Anesthesiology. 2005 ;103:258-68.

13. Shimoyama M, Shimoyama N, Gorman A, Elliot R, Inturrici C. Oral Ketamine is antinociceptive in the rat: the role of norketamine. Pain 1999; 81: 85-93.

14. Krystal J, Karper L, Benett A. Interactive effects of subanestetic ketamine and subhypnotic lorazepam in humans. Psichopharmacology 1998; 38: 203-19.

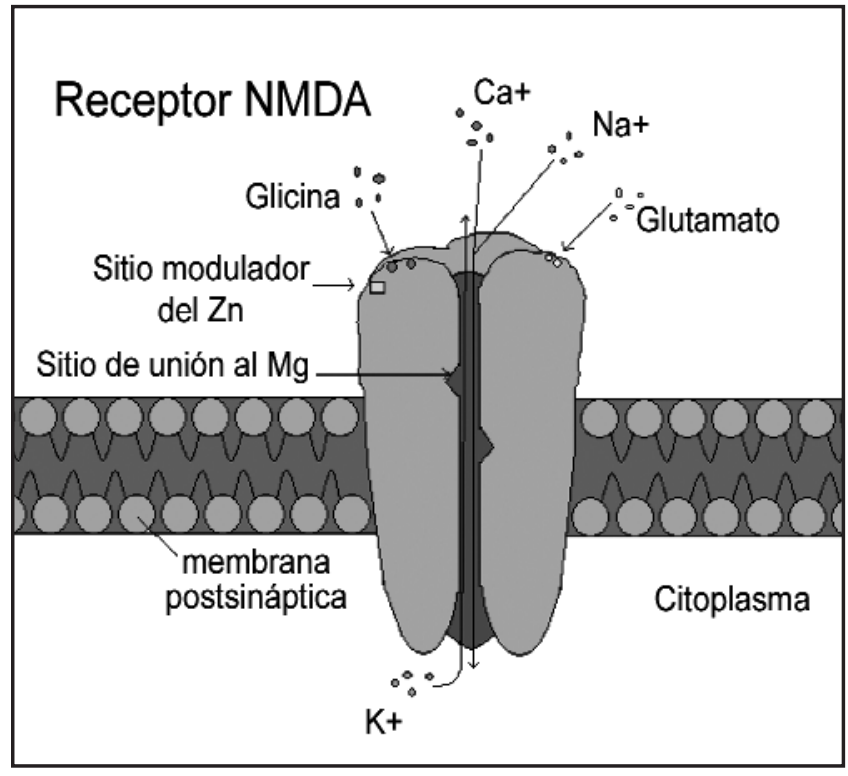

Figura 1. Receptor NMDA. Modificado de www.SNCforum.com

15. Fisher K, Coderre T, Hagen N. Targeting the N-Methyl-D-Aspartate receptor for chronic pain management: preclinical animal studies, recent clinical experience and future research directions. J Pain Symptom Manage 2000; 20:358-73.

16. Annetta M, Iemma D, Garisto C, Tafani C, Proietti R. Ketamine: new indications for an old drug. Curr Drug Targets. 2005;6:789-94.

17. Lynch M, Clark A, Sawynok J, Sullivan M. Topical amitriptyline and ketamine in neuropathic pain syndromes: an open-label study. J Pain. $2005 ; 6: 644-9$

18. Reves J, et al. Intravenous nonopioid anesthetics. In: Miller R, editor. Miller's anesthesia. Philadelphia: Hanley and Belfus; 2005. p. 317-78.

19. Larcher A, Laulin J, Celerier E, Le Moal M, Simonnet G. Acute tolerance associated with a single opiate administration: involvement of N-methyl-D-aspartate-dependent pain facilitatory systems. Neuroscience 1998; 84:583-9.

20. Akin Takmaz S, Inan N, Gunal S, Kaymak C, Sakalli M, Dikmen B. Ketamine combined with morphine for the management of cancer pain in a patient with meperidine tolerance and addiction. Agri. 2005 Jul;17:44-7.

21. Anghelescu DL, Oakes LL. Ketamine use for reduction of opioid tolerance in a 5-year-old girl with end-stage abdominal neuroblastoma. J Pain Symptom Manage. 2005;30:1-3.

22. Fitzgibbon EJ, Viola R. Parenteral ketamine as an analgesic adjuvant for severe pain: development and retrospective audit of a protocol for a palliative care unit. J Palliat Med. 2005;8:49-57.

23. Lossignol DA, Obiols-Portis M, Body JJ. Successful use of ketamine for intractable cancer pain. Support Care Cancer. 2005;13:188-93.

24. Slatkin NE, Rhiner M. Ketamine in the treatment of refractory cancer pain: case report, rationale, and methodology. J Support Oncol. 2003;1:287-93

25. Kotlinska-Lemieszek A, Luczak J. Subanesthetic ketamine: an essential adjuvant for intractable cancer pain. J Pain Symptom Manage. 2004;28:100-2

26. Rae F, Eccleston C, Kalso E. Ketamine as adjuvant to opioids for cancer pain. A Qualitative Systematic Review. J Pain Symptom Manage 2003; 26:867-875 
27. Thogulava R, Saxena A., Sushma B. Oral Ketamine as an adjuvant to oral morphine for neuropathic pain in cancer patients. J Pain Symptom Manage. 2002;23:60-65

28. Kannan T. Oral ketamine as an adjuvant to oral morphine for neuropathic pain in cancer patients. J Pain Symptom Manage 2002;23:60-5.

29. Friedman R., Li V., Keuppers F., Atkinson G. Oral Ketamine in hepatocellular carcinoma. Pain medicine 2000; 1: 181-182.

30. McDonnell F., Sloan J., Hamann S: Advances in cancer pain management. Current Pain Headache Rep 5: 265-71, 2001.

31. Ban C,Tsui H, Dawn D, Sunil D, Malherbe S, Intravenous Ketamine Infusion as an Adjuvant to Morphine in a 2-Year-Old With Severe Cancer Pain From Metastatic Neuroblastoma. J Pediatr Hematol Oncol. 2004;26:678-680.

32. Mercadante S, Arcuri E, Tirelli W. Analgesic Effect of Intravenous Ketamine in Cancer Patients on Morphine Therapy: A Randomized, Controlled, Double-Blind, Crossover, Double-Dose Study. J Pain Symptom Manage. 2000; 20:246-252.

33. Yang $\mathrm{C}$, Wong $\mathrm{C}$, Chang $\mathrm{J}$. Intrathecal ketamine reduces morphine requirements in patients with terminal cancer pain. Canadian $\mathrm{J}$ of Anaesthesia 1996; 43:379-83.

34. Carr D, Goudas L, Denmana W, BroOkoff D, Staats P, Brennen L, Green G, Albine R, Hamilton D, Rogers M, Firestone L, Alvin R, Mermelstein F. Safety and efficacy of intranasal ketamine for the treatment of breakthrough pain in patients with chronic pain: a randomized,double-blind, placebo-controlled, crossover study. Pain 2004;108: 17-27.

35. Gammaitoni A, Gallagher R, Welz-Bosna M. Topical ketamine gel: possible role in treating neuropathic pain. Pain Med. 2000;1:97-100.

36. Yaster M. General anesthesia. In: Yaster M, et al., editors. Pediatric pain management and sedation handbook. St. Louis: MosbyYearbook; 1997. p. 376 